\title{
Prognostic Role and Clinical Association of Tumor-Infiltrating Lymphocyte, Programmed Death Ligand-1 Expression with Neutrophil- Lymphocyte Ratio in Locally Advanced Triple-Negative Breast Cancer
}

\author{
Jieun Lee, $M D, P h D^{1,2}$ \\ Dong-Min Kim, BS 3 \\ Ahwon Lee, MD, $\mathrm{PhD} 2,3$
}

\begin{abstract}
Purpose
Tumor-infiltrating lymphocyte (TIL), programmed death-ligand 1 (PD-L1) expression and neutrophil-to-lymphocyte ratio (NLR) is associated to immunogenicity and prognosis of breast cancer. We analyzed baseline NLR, changes of NLR, TIL, and PD-L1 during neoadjuvant chemotherapy (NAC) and their clinical implication in triple-negative breast cancer (TNBC).
\end{abstract}

\section{Materials and Methods}

Between January 2008 to December 2015, 358 TNBC patients were analyzed. Baseline NLR, 50 paired NLR (initial diagnosis, after completion of NAC) and 34 paired tissues (initial diagnosis, surgical specimen after completion of NAC) were collected. Changes of TIL, CD4, CD8, forkhead box P3 (FOXP3), and PD-L1 expression were assessed with immunohistochemical stain.

${ }^{1}$ Division of Medical Oncology, Department of Internal Medicine, College of Medicine, The Catholic University of Korea, Seoul, ${ }^{2}$ Cancer Research Institute, The Catholic University of Korea, Seoul, ${ }^{3}$ Department of Hospital Pathology, College of Medicine, The Catholic University of Korea, Seoul, Korea
Correspondence: Ahwon Lee, MD, PhD Department of Hospital Pathology, College of Medicine, The Catholic University of Korea, 222 Banpo-daero, Seocho-gu, Seoul 06591, Korea

Tel: 82-2-2258-1621

Fax: 82-2-2258-1627

E-mail: klee@catholic.ac.kr

Received May 10, 2018

Accepted July 26, 2018

Published Online July 31, 2018

\section{Key words}

Triple-negative breast neoplasms, Neutrophil-lymphocyteratio, Tumor-infiltrating lymphocytes, PD-L1

\section{Introduction}

Triple-negative breast cancer (TNBC) accounts for 10 to $20 \%$ of total breast cancer and is defined as absence of estrogen receptor (ER), progesterone receptor (PR), and human epidermal growth factor-2 (HER-2) expression by immunohistochemical stain. Compared to other subtypes, TNBC shows relatively poorer outcome due to its aggressive clinical behavior and lack of optimal molecular target for effective treatment [1]. Molecular profiling may more accurately predict the prognosis and response to chemotherapy of TNBC [2], but genomic profiling is not routinely feasible in clinical setting [3]. Other than genomic analysis, various clinical, laboratory and pathologic parameters are assessed for convenient prediction of chemotherapy response and overall prog- 
nosis of TNBC.

Inflammation is traditionally known to be associated with oncogenesis and progression in various malignancies. Systemic inflammation and local immune response both reflect prognosis of many solid cancers, and cross-talk between two immune response is suspected in progression of malignancies [4]. Neutrophil-lymphocyte ratio (NLR) represents systemic inflammation, and there are various reports supporting NLR reflects prognosis in solid cancers [4], including breast cancer $[3,5,6]$. In locally advanced breast cancer, pretreatment NLR is reported to be associated with superior response to neoadjuvant chemotherapy (NAC) and diseasefree survival (DFS) [3,7]. Tumor-infiltrating lymphocyte (TIL) is suggested to represent anti-tumor immune response in tumor environment and associated with prognosis in various cancers including breast cancer [8-10]. In breast cancer patients who receive NAC, the presence of TIL and the changes of $\mathrm{T}$ cell marker is reported to be associated to patients' prognosis [11]. Programmed cell death receptor 1 (PD-1), programmed cell death receptor ligand-1 (PD-L1) is one of a major molecule regulating T-cell activation and immune surveillance [12]. PD-L1 is expressed in both tumor cell and stroma, with heterogeneous distribution between cancers, including breast cancer [13]. PD-L1 expression is reported to be correlated to TIL and response to NAC in breast cancer [14] and other literatures report that expression of PD-L1 may be associated to prognosis in breast cancer, but with conflicting results $[15,16]$.

The association of NLR, TIL, or PD-L1 with clinicopathologic parameters or survival outcomes were previously studied in various cancers $[5-7,9,10,17]$. NLR, TIL, and PD-L1 expression in breast cancer each has been studied as predictive or prognostic marker for estimating the response of NAC, DFS, or overall survival (OS) in various literatures $[3,7,14,17]$. Among NLR, TIL, and PD-L1, the role or NLR was most extensively studied in breast cancer, but the patient population was heterogeneous with mixture of subtypes or commonly studied in hormone receptor positive breast cancer $[3,6,7]$. The expression or changes of TIL during chemotherapy is traditionally known to be associated to tumor response in breast cancer [18], and the association between TIL and PD-L1 expression among tumor tissues are recently updated, but with heterogeneous reports and analyses methods $[14,17,19,20]$. Additionally, the role of PD-L1 in predicting prognosis and tumor response in breast canceris reported with conflicting results $[20,21]$. The interaction between systemic inflammatory marker such as NLR and local inflammatory marker such as TIL or PD-L1 has not been studied in-depth previously. Immunogenicity and related tumor response and prognosis is becoming more important in breast cancer, and administration of NAC may potentiate the changes of immunogenicity in breast cancer population.
Therefore, we investigated the role of NLR, TIL, and PD-1 in homogeneous TNBC patient population.

In this study, we primarily analyzed the association of NLR with survival outcomes in early or locally advanced TNBC. Furthermore, the association between clinical outcomes, TIL and PD-L1 was analyzed in subgroup of patients who received NAC. Interrelationship between NLR, TIL, $\mathrm{CD}^{+}$TIL, CD8 ${ }^{+} \mathrm{TIL}$, forkhead box P3 (FOXP3)+ $\mathrm{TIL}$, and PDL1 expression was also studied among NAC patients.

\section{Materials and Methods}

\section{Patients}

From January 2007 to December 2015, the medical records of 358 patients who were diagnosed as TNBC in Seoul St. Mary's Hospital were retrospectively reviewed. All patients were pathologically diagnosed as stage I to III and received adequate local, systemic treatment. Triple negative breast cancer was defined as ER, PR, and HER2 all negative by American Society of Clinical. Oncology-College of American Pathologists guideline [22,23]; ER and PR negative defined as $<1 \%$ tumor cells displaying nuclear staining. HER2 negativity defined as HER2 immunohistochemistry 0/1 (no staining, faint/barely perceptible membrane staining, or weak incomplete membrane staining in $<10 \%$ of invasive tumor cells) or HER2 silver in situ hybridization negative (Dual-probe HER2/Chr17 ratio < 2.0 with an average HER2 copy number $<4.0$ signals / cell). Among 358 patients, 50 patients received NAC. Baseline complete blood count (CBC) was collected before surgical treatment. The other eligible criteria were as follows: (1) adequate bone marrow function without hematologic disease; (2) patients who regularly followed up in Seoul St. Mary's Hospital. In 50 patients who received NAC, baseline $\mathrm{CBC}$ was collected at initial pathologic diagnosis, and $\mathrm{CBC}$ was recollected before patient went through surgical treatment after completion of NAC. Among 34 patients who received $\mathrm{NAC}$, baseline $\mathrm{CBC}$, pre-operative $\mathrm{CBC}$, biopsy specimen obtained during initial diagnosis and surgical pathologic specimens were collected.

\section{Treatments}

Initial pathologic diagnosis, laboratory evaluation and staging were done within 2 weeks since initial patient visit. In patient population who received primary breast conserving surgery or total mastectomy with axillary node staging, they received adequate anthracycline or taxane based adjuvant chemotherapy or standard CMF regimen and radiation 
therapy. Adjuvant chemotherapy regimens are as follows: (1) adriamycin $\left(60 \mathrm{mg} / \mathrm{m}^{2}\right.$ intravenously, day 1$)$ and cyclophosphamide (600 $\mathrm{mg} / \mathrm{m}^{2}$ intravenously, day 1 ) every 3 weeks for 4 cycles followed by docetaxel $\left(75 \mathrm{mg} / \mathrm{m}^{2}\right.$ intravenously, day 1) every 3 weeks for 4 cycles (AC-T); (2) adriamycin $\left(60 \mathrm{mg} / \mathrm{m}^{2}\right.$ intravenously, day 1$)$ and cyclophosphamide $\left(600 \mathrm{mg} / \mathrm{m}^{2}\right.$ intravenously, day 1 ) (AC) every 3 weeks for 4 cycles; (3) docetaxel (75 $\mathrm{mg} / \mathrm{m}^{2}$ intravenously, day 1) and adriamycin $\left(50 \mathrm{mg} / \mathrm{m}^{2}\right.$ intravenously, day 1$)$ (DA) every 3 weeks for $2-4$ cycles; (4) docetaxel $\left(75 \mathrm{mg} / \mathrm{m}^{2}\right.$ intravenously, day 1) and cyclophosphamide $\left(600 \mathrm{mg} / \mathrm{m}^{2}\right.$ intravenously, day 1) (TC) every 3 weeks for 4 cycles; (5) docetaxel $\left(75 \mathrm{mg} / \mathrm{m}^{2}\right.$ intravenously, day 1$)$, adriamycin $\left(50 \mathrm{mg} / \mathrm{m}^{2}\right.$ intravenously, day 1), and cyclophosphamide $\left(500 \mathrm{mg} / \mathrm{m}^{2}\right.$ intravenously, day 1) (TAC) every 3 weeks for 6 cycles; (6) 5-fluorouracil (5-FU; $600 \mathrm{mg} / \mathrm{m}^{2}$ intravenously, day 1), epirubiclin $\left(60 \mathrm{mg} / \mathrm{m}^{2}\right.$ intravenously, day 1$)$, and cyclophosphamide $\left(600 \mathrm{mg} / \mathrm{m}^{2}\right.$ intravenously, day 1) (FEC); (7) oral cyclophosphamide (100 mg/m² intravenously, day 1 to 14 ), methotrexate ( $40 \mathrm{mg} / \mathrm{m}^{2}$ intravenously, day 1 and 8), and 5-FU (600 mg/m² IV, day 1 and 8) (CMF) every 4 weeks for 6 cycles. Among patients who were diagnosed as stage II or III breast cancer, part of patients received anthracycline and taxane based NAC followed by surgery and adjuvant radiation. Anthracycline and taxane based NAC regimen is as follows: (1) docetaxel $\left(75 \mathrm{mg} / \mathrm{m}^{2}\right.$ intravenously, day 1$)$ and adriamycin (50 mg/m² intravenously, day 1) (DA) every 3 weeks for $2-6$ cycles; (2) adriamycin $\left(60 \mathrm{mg} / \mathrm{m}^{2}\right.$ intravenously, day 1) and cyclophosphamide $\left(600 \mathrm{mg} / \mathrm{m}^{2}\right.$ intravenously, day 1) (AC) every 3 weeks for 4 cycles; (3) epirubicin $\left(75 \mathrm{mg} / \mathrm{m}^{2}\right.$ intravenously, day 1$)$ and cyclophosphamide $\left(600 \mathrm{mg} / \mathrm{m}^{2}\right.$ intravenously, day 1 ) (EC) for 4 cycles. Patients were followed by every 3 months with history taking and physical examination. Laboratory evaluation including serum cancer antigen 15-3 and carcinoembryonic antigen, chest and abdominal computed tomography (CT) scan were done if clinically warranted. Mammography and breast sonography was done every six months for follow-up (S1 Fig.).

\section{Definition of neutrophil-to-lymphocyte ratio}

Neutrophil-to-lymphocyte (NLR) ratio was determined as ratio of neutrophil count to lymphocyte in CBC. Among patients who received NAC, baseline NLR was collected during initial diagnosis and post-NAC NLR was collected before breast surgery. Patients who initially received breast surgery, NLR was collected before surgical treatment. The cutoff value of NLR was decided as 3.16, which was the maximum (sensitivity+specificity) point according to receiver operating characteristic (ROC) curve. ROC curve was analyzed with OS data of total patient population. In patients who received NAC, radical NLR change was defined as $<-30 \%$ or $>100 \%$ based on quartiles of NLR change distribution.
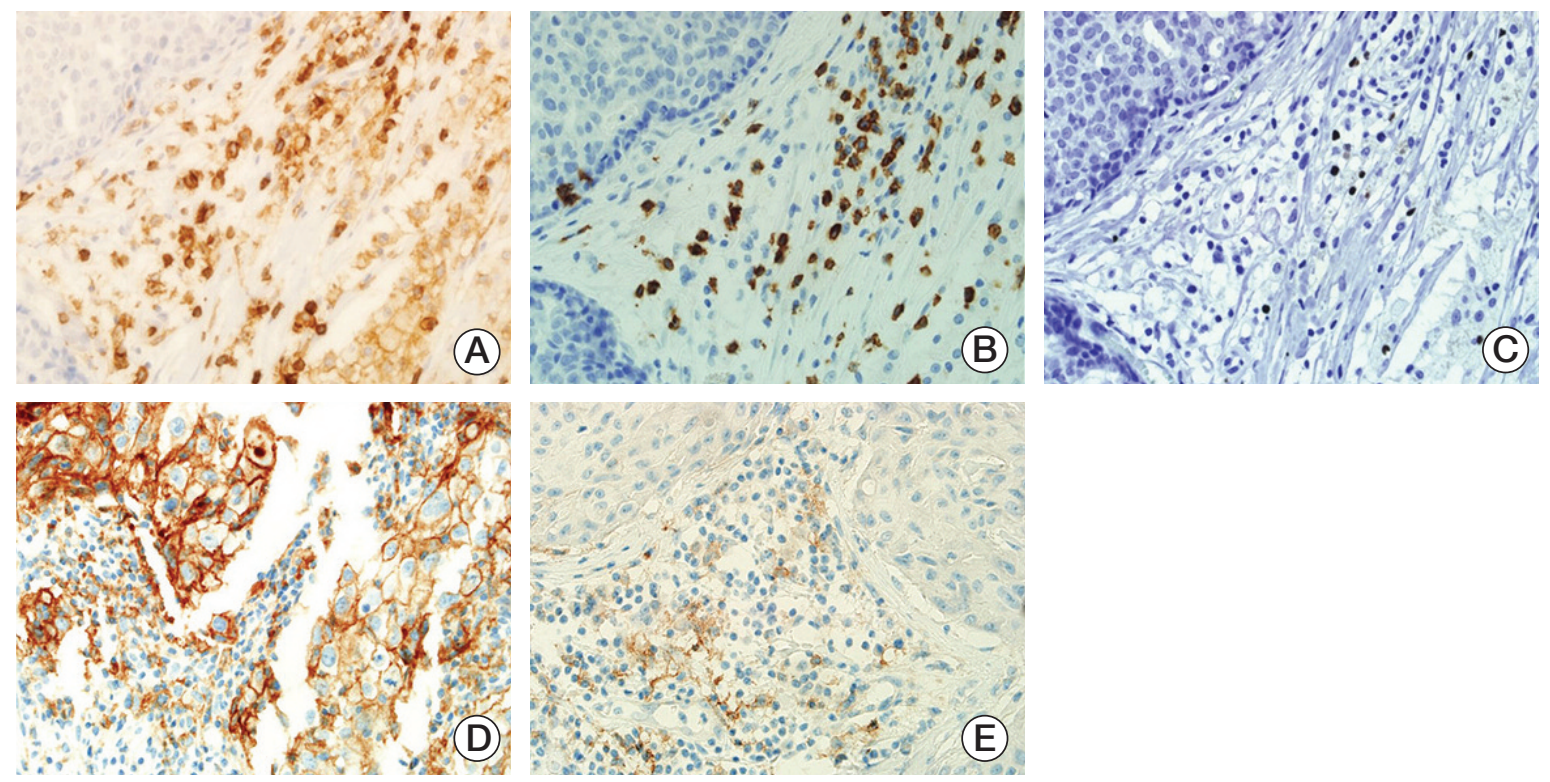

Fig. 1. Representative staining of tumor infiltrating-lymphocytes (TILs) and programmed death ligand-1 (PD-L1) expression in tumor specimens. (A) CD4 ${ }^{+}$TILs ( $\left.\times 400\right)$. (B) CD8 ${ }^{+}$TILs $(\times 400)$. (C) Forkhead box P3 ${ }^{+}$TILs $(\times 400)$. (D) PD-L1 ( $\times 400$, H-score 200). (E) PD-L1 ( $\times 400$, H-score 75$)$. 
Table 1. Baseline patient characteristics

\begin{tabular}{|c|c|c|c|c|}
\hline & Total & $\mathrm{NLR} \leq 3.16$ & NLR $>3.16$ & p-value \\
\hline No. of patients & 358 & $313(87.4)$ & $45(12.6)$ & \\
\hline NLR & $1.76(0.12-10.06)$ & $1.64(0.12-3.16)$ & $3.95(3.2-10.06)$ & \\
\hline \multicolumn{5}{|l|}{ Age (yr) } \\
\hline Median (range) & $51(14-86)$ & $51(14-86)$ & $48(32-83)$ & 0.970 \\
\hline$<40$ & $71(19.8)$ & $62(17.3)$ & $9(2.5)$ & \\
\hline$\geq 40$ & $287(80.2)$ & $251(70.1)$ & $36(10.1)$ & \\
\hline \multicolumn{5}{|l|}{ Stage } \\
\hline I & $127(35.5)$ & $113(31.6)$ & $14(3.9)$ & 0.340 \\
\hline II & $164(45.8)$ & $145(40.5)$ & $19(5.3)$ & \\
\hline III & $67(18.7)$ & $55(15.3)$ & $12(3.4)$ & \\
\hline \multicolumn{5}{|l|}{ Histology } \\
\hline Invasive ductal carcinoma & $300(83.8)$ & $260(72.6)$ & $40(11.2)$ & 0.460 \\
\hline Invasive lobular carcinoma & $6(1.7)$ & $6(1.7)$ & 0 & \\
\hline Medullary carcinoma & $17(4.7)$ & $14(3.9)$ & $3(0.8)$ & \\
\hline Metaplastic carcinoma & $17(4.7)$ & $16(4.4)$ & $1(0.3)$ & \\
\hline Others & $4(1.1)$ & $4(1.1)$ & 0 & \\
\hline \multicolumn{5}{|l|}{ Histologic grade } \\
\hline Well-moderately differentiated & $103(28.8)$ & $86(24.0)$ & $17(4.8)$ & 0.408 \\
\hline Poorly differentiated & $252(70.4)$ & $224(62.6)$ & $28(7.8)$ & \\
\hline Unknown & $3(0.8)$ & $3(0.8)$ & 0 & \\
\hline \multicolumn{5}{|l|}{ Ki-67 } \\
\hline$<20 \%$ & $48(13.4)$ & $43(12.0)$ & $5(1.4)$ & 0.677 \\
\hline$\geq 20 \%$ & $303(84.6)$ & $265(74.0)$ & $38(10.6)$ & \\
\hline Unknown & $7(2.0)$ & $5(1.4)$ & $2(0.6)$ & \\
\hline Adjuvant chemotherapy & $310(86.6)$ & $271(75.7)$ & $39(10.9)$ & 0.540 \\
\hline AC followed by docetaxel & $64(17.9)$ & $59(16.4)$ & $5(1.5)$ & \\
\hline $\mathrm{AC}$ & $171(47.8)$ & $149(41.6)$ & $22(6.2)$ & \\
\hline DA & $14(3.9)$ & $8(2.2)$ & $6(1.7)$ & \\
\hline CMF & $47(13.1)$ & $42(11.7)$ & $5(1.4)$ & \\
\hline $\mathrm{TC}$ & $11(3.1)$ & $10(2.8)$ & $1(0.3)$ & \\
\hline TAC & $2(0.6)$ & $2(0.6)$ & 0 & \\
\hline FEC & $1(0.3)$ & $1(0.3)$ & 0 & \\
\hline \multicolumn{5}{|l|}{ Adjuvant radiation } \\
\hline Yes & $267(74.6)$ & $234(65.4)$ & $33(9.2)$ & 0.900 \\
\hline No & $91(25.4)$ & $79(22.1)$ & $12(3.3)$ & \\
\hline Cancer recurrence & $47(13.1)$ & $37(10.3)$ & $10(2.8)$ & 0.047 \\
\hline Death & $36(10.1)$ & $26(7.3)$ & $10(2.8)$ & 0.004 \\
\hline
\end{tabular}

Values are presented as median (range) or number (\%). NLR, neutrophil-to-lymphocyte ratio; AC, adriamycin-cyclophosphamide; DA, docetaxel-adriamycin; CMF, cyclophosphamide-methotrexate-5-fluorouracil; TC, docetaxel-cyclophosphamide; TAC, docetaxel-adriamycin-cyclophosphamide; FEC, 5-fluorouracil-epirubicin-cyclophosphamide.

\section{Immunohistochemical staining and pathologic analysis}

All 34 pathologic tissue specimens including diagnostic biopsy tissues and surgical specimens were formalin-fixed and stored as paraffin blocks in the Department of Hospital Pathology, Seoul St. Mary's Hospital, The Catholic University of Korea. Paraffin blocks were cut into $4-\mu \mathrm{m}$ sections. Pri- mary antibodies were used for immunohistochemical staining: anti-CD4 rabbit monoclonal (clone SP35, Ventana Medical Systems, Tucson, AZ), anti-CD8 mouse monoclonal (clone C8/144B, DAKO, Glostrup, Denmark), anti-FOXP3 mouse monoclonal (clone 236A/E7, 1:50 dilution, Abcam, Cambridge, UK), and anti-PD-L1 rabbit monoclonal (clone SP263, Ventana Medical Systems). The sections were deparaffinized 
Table 2. Baseline characteristics of patients receiving neoadjuvant chemotherapy

\begin{tabular}{|c|c|c|c|c|}
\hline & Total & $\mathrm{NLR} \leq 3.16$ & $\mathrm{NLR}>3.16$ & p-value \\
\hline No. of patients & 50 & $42(84)$ & $8(16)$ & 0.430 \\
\hline \multicolumn{5}{|l|}{ Age (yr) } \\
\hline Median (range) & & $43.5(25-66)$ & $43.5(36-51)$ & 0.600 \\
\hline$<40$ & $15(30)$ & $13(26)$ & $2(4)$ & \\
\hline$\geq 40$ & $35(70)$ & $29(58)$ & $6(12)$ & \\
\hline \multicolumn{5}{|l|}{ Clinical stage } \\
\hline II & $9(18)$ & $9(18)$ & 0 & 0.150 \\
\hline III & $41(82)$ & $33(66)$ & $8(16)$ & \\
\hline \multicolumn{5}{|l|}{ Pathologic stage } \\
\hline 0 & $3(6)$ & $3(6)$ & 0 & 0.260 \\
\hline I & $7(14)$ & $6(12)$ & $1(2)$ & \\
\hline II & $16(32)$ & $12(24)$ & $4(8)$ & \\
\hline III & $14(28)$ & $11(22)$ & $3(6)$ & \\
\hline \multicolumn{5}{|l|}{ Downstaging } \\
\hline No & $6(12)$ & $4(8)$ & $2(4)$ & 0.500 \\
\hline Yes & $44(88)$ & $38(76)$ & $6(12)$ & \\
\hline pCR & $5(10)$ & $5(10)$ & 0 & 0.300 \\
\hline \multicolumn{5}{|c|}{ Neoadjuvant chemotherapy } \\
\hline $\mathrm{AC}$ & $13(26)$ & $11(22)$ & $2(4)$ & 0.870 \\
\hline $\mathrm{EC}$ & $5(10)$ & $5(10)$ & 0 & \\
\hline DA & $32(64)$ & $26(52)$ & $6(12)$ & \\
\hline \multicolumn{5}{|l|}{ Adjuvant radiation } \\
\hline Yes & $36(72)$ & $29(58)$ & $7(14)$ & 0.400 \\
\hline No & $14(28)$ & $13(26)$ & $1(2)$ & \\
\hline Cancer recurrence & $9(18)$ & $6(12)$ & $3(6)$ & 0.500 \\
\hline Death & $8(16)$ & $6(12)$ & $2(4)$ & 0.450 \\
\hline
\end{tabular}

Values are presented as number $(\%)$ unless otherwise indicated. NLR, neutrophil-to-lymphocyte ratio; pCR, pathologic compete response; AC, adriamycin-cyclophosphamide; EC, epirubicin-cyclophosphamide; DA, docetaxel-adriamycin.

in xylene for 15 minutes, subsequently rehydrated and rinsed in distilled water. For CD3, CD4, and PD-L1, assays were then performed using the Ventana NX automated immunohistochemistry system (Ventana Medical Systems). For FOXP3 immunohistochemical staining, antigen retrieval was carried out in a pressure cooker (Electric Pressure Cooker CPC-600, Cuisinart, East Windsor, NJ) for 20 minutes using $1 \times$ citrate buffer ( $\mathrm{pH}$ 6.0). The endogenous peroxide activity was blocked by methanol-diluted 3\% hydrogen peroxide for 15 minutes. Sections were incubated with primary antibodies for 1 hour at room temperature in a humidified chamber. The signal of immunoreaction was amplified and revealed using Polink-2 plus HRP Rabbit DAB kit (GBI, Mukilteo, WA). Subsequently, these slides were counterstained with Harris's hematoxylin. Histopathologic evaluations for $\mathrm{CD}^{+}, \mathrm{CD}^{+}$, and $\mathrm{FOXP}^{+}{ }^{+}$TILs were performed by senior pathologist who was not aware of patient information. Each full-face tumor section slide was evaluated for stromal TIL using microscopic examination $(\times 400)$. Five independent sections with- out massive inflammation or necrosis were selected and photographed by Olympus DP72 camera (Tokyo, Japan). The mean absolute number of $\mathrm{CD}^{+}, \mathrm{CD} 8^{+}$, and $\mathrm{FOXP} 3^{+}$TILs per square millimeter was reviewed by two individual investigators. Positive PD-L1 expression was evaluated within the boundary of invasive cancer. PD-L1 expression in tumor cells and stroma were studied and graded by percentage of positive cells and the staining intensity (1-3). An H-score (percentage of positive cells $\times$ staining intensity, ranging $0-300$ ) was used for PD-L1 expression analysis, and the cut-off value was determined by $\mathrm{H}$-score of 5, based on the reference [20]. The representative sections are described in Fig. 1.

\section{Statistical analysis}

OS was determined as from the pathologic diagnostic date to the last follow-up date or patient's death. DFS was defined as from the date of primary surgery to the date of cancer recurrence, proven by imaging study such as chest or abdo- 

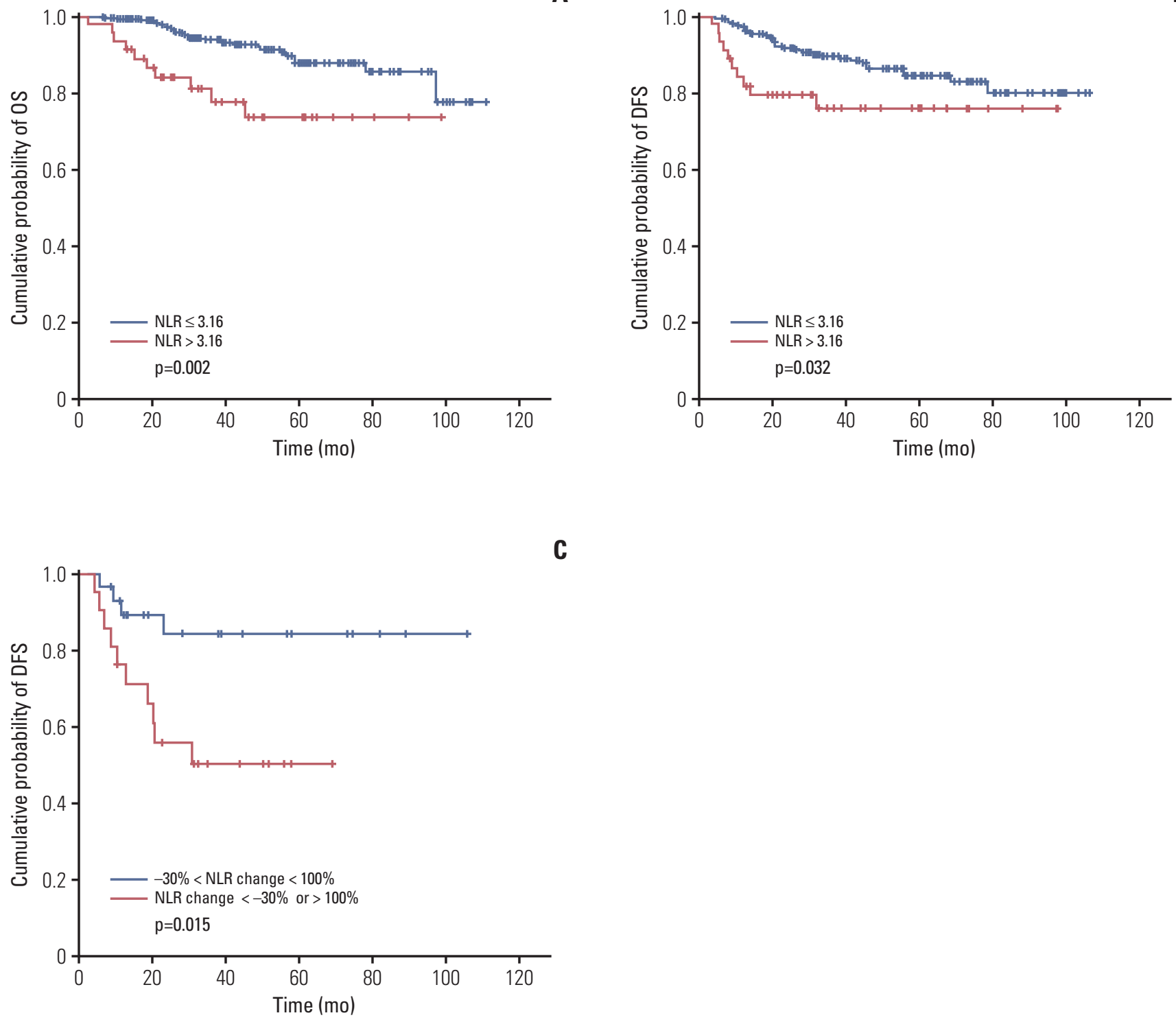

C

Fig. 2. (A) Overall survival (OS) of patients according to neutrophil-lymphocyte ratio (NLR) level. (B) Disease-free survival of patients according to NLR level. (C) Disease-free survival (DFS) of patients who received neoadjuvant chemotherapy according to changes of NLR.

men CT scans. OS and DFS were analyzed using log-rank test and Kaplan-Meier method. Cutoff value of NLR was decided according to maximum (sensitivity+specificity) point of ROC curve. Comparison between changes among baseline and post-neoadjuvant parameters were compared using Mann-Whitney U test. NLR, TILs, and PD-L1 expressions among patients were compared using spearman correlation analysis. Statistical analyses were performed using SPSS ver. 24.0 software (IBM Corp., Armonk, NY).

\section{Ethical statement}

This study was approved by the Institutional Review Board (IRB) of Seoul St. Mary's Hospital, The Catholic University of Korea (KC17EESI0084). The requirement for written informed consent was waived according to the decision of IRB. 
Table 3. Univariate and multivariate analysis of disease-free survival and overall survival

\begin{tabular}{|c|c|c|c|c|c|c|c|c|}
\hline \multirow{3}{*}{ Characteristic } & \multicolumn{4}{|c|}{ Overall survival } & \multicolumn{4}{|c|}{ Disease-free survival } \\
\hline & \multicolumn{2}{|c|}{ Univariate analysis } & \multicolumn{2}{|c|}{ Multivariate analysis } & \multicolumn{2}{|c|}{ Univariate analysis } & \multicolumn{2}{|c|}{ Multivariate analysis } \\
\hline & HR $(95 \%$ CI) & p-value & HR $(95 \%$ CI) & p-value & HR $(95 \%$ CI) & p-value & HR $(95 \%$ CI $)$ & p-value \\
\hline \multicolumn{9}{|l|}{ Age (yr) } \\
\hline$\geq 40$ vs. $<40$ & $1.167(0.6-2.67)$ & 0.720 & - & - & $0.83(0.42-1.63)$ & 0.580 & - & - \\
\hline \multicolumn{9}{|l|}{ Stage } \\
\hline I & 1 (reference) & $<0.001$ & 1 (reference) & 0.114 & 1 (reference) & $<0.001$ & 1 (reference) & $<0.001$ \\
\hline II vs. I & $2.13(0.84-5.45)$ & 0.110 & $3.15(1.03-9.68)$ & 0.045 & $1.41(0.67-2.97)$ & 0.361 & $1.33(0.61-2.95)$ & 0.470 \\
\hline III vs. I & $5.97(2.23-15.6)$ & $<0.001$ & $3.12(0.96-10.01)$ & 0.058 & $4.07(1.91-8.72)$ & $<0.001$ & $4.0(1.81-8.85)$ & 0.001 \\
\hline \multicolumn{9}{|l|}{ NLR } \\
\hline$>3.16$ vs. $\leq 3.16$ & $2.97(1.43-6.17)$ & 0.003 & $3.15(1.3-7.5)$ & 0.009 & $2.11(1.05-4.25)$ & 0.036 & $1.73(0.83-3.6)$ & 0.140 \\
\hline \multicolumn{9}{|l|}{$\mathrm{Ki}-67(\%)$} \\
\hline$\geq 20$ vs. $<20$ & $3.11(0.74-13.1)$ & 0.120 & $3.68(0.87-15.7)$ & 0.070 & $1.003(0.44-2.26)$ & 0.994 & - & - \\
\hline \multicolumn{9}{|c|}{$\begin{array}{l}\text { History of adjuvant } \\
\text { chemotherapy }\end{array}$} \\
\hline Yes vs. no & $1.57(0.48-5.14)$ & 0.460 & - & - & $2.18(0.68-7.04)$ & 0.190 & - & - \\
\hline \multicolumn{9}{|l|}{ Cancer recurrence } \\
\hline Yes vs. no & $55.9(19.7-159.2)$ & $<0.001$ & $58.2(19.76-171.4)$ & $<0.001$ & - & - & - & - \\
\hline
\end{tabular}

$\mathrm{HR}$, hazard ratio; CI, confidence interval; NLR, neutrophil-to-lymphocyte ratio.

\section{Results}

\section{Patient characteristics}

Between January 2007 to December 2015, total 358 patients who were diagnosed as TNBC were enrolled for study. Baseline patient characteristics are described in Table 1. The median age of total patient population was 51 years. More than half of patients (231 patients, 64.5\%) were diagnosed as stage II or III TNBC at initial diagnosis. Eighty-seven percent of total patients received adjuvant chemotherapy, and most of them received anthracycline or taxane based chemotherapy. Among total patient population, $13.1 \%$ of patients experienced cancer recurrence and $10.1 \%$ of total patients died due to breast cancer. The median NLR of total patients were 1.76. Forty-five patients showed high NLR (NLR > 3.16), and these patients showed higher cancer recurrence and mortality compared to low NLR patient group (Table 1).

Fifty patients $(13.9 \%)$ received NAC before surgical treatment. The baseline characteristics of patients who received NAC is described in Table 2. Ten percent among 50 patients showed pathologic complete response ( $\mathrm{pCR}$ ) after neoadjuvant chemotherapy, and rest of patients received additional adjuvant chemotherapy after surgery. Among 50 patients, $18 \%$ of patients showed cancer recurrence and $16 \%$ of patients showed cancer-related death. Patients with high NLR (8 patients, 16\%) were diagnosed as stage III TNBC, and no patients showed $\mathrm{pCR}$ after NAC. The distribution pattern of NLR in total population, and changes of NLR during NAC is depicted as follows (S2 Fig.).

\section{Association between NLR and survival outcomes}

In total patient population, patients with low NLR $(\leq 3.16)$ showed longer OS and DFS compared to high NLR (>3.16) group. Low NLR patient group showed superior OS (median, 41.83 months; 95\% confidence interval [CI], 6.13 to not estimable) compared to high NLR group (median OS, 36.5 months; $95 \%$ CI, 2.37 to not estimable), with statistical significance $(p=0.002)$ (Fig. 2A). Similarly, patients with low NLR showed longer DFS (median, 37.85 months; 95\% CI, 3.93 to not estimable) compared to patients with high NLR (median, 32.14 months; 95\% CI, 3.37 to not estimable), with statistical significance ( $p=0.032)$ (Fig. 2B).

Among patients who received NAC, lower baseline NLR patient group showed trends for superior OS and DFS compared to higher baseline NLR patient group, but without statistical significance (data not shown). Comparing the change between baseline NLR and post-NAC NLR in patients who received NAC, patients who showed radical change of NLR (NLR change $<-30 \%$ or $>100 \%$ ) showed inferior DFS compared to patients who showed relatively small NLR changes during NAC (median DFS, 38.37 months vs. 22.37 months; $\mathrm{p}=0.015)$ (Fig. 2C). 
Table 4. Univariate and Multivariate analysis of disease-free survival in neoadjuvant patients

\begin{tabular}{|c|c|c|c|c|}
\hline \multirow{2}{*}{ Characteristic } & \multicolumn{2}{|c|}{ Univariate analysis } & \multicolumn{2}{|c|}{ Multivariate analysis } \\
\hline & Hazard ratio $(95 \% \mathrm{CI})$ & $\overline{p \text {-value }}$ & Hazard ratio $(95 \% \mathrm{CI})$ & p-value \\
\hline \multicolumn{5}{|l|}{ Age (yr) } \\
\hline$<40$ vs. $\geq 40$ & $1.07(0.33-3.42)$ & 0.900 & - & - \\
\hline \multicolumn{5}{|l|}{ Clinical stage } \\
\hline II vs. III & $3.76(0.49-28.85)$ & 0.200 & - & - \\
\hline Surgical stage & & 0.034 & & 0.027 \\
\hline 0 vs. I & $0.51(0.046-5.65)$ & 0.590 & $0.37(0.33-4.24)$ & 0.430 \\
\hline 0 vs. II & $0.5(0.052-4.83)$ & 0.550 & $0.39(0.4-3.84)$ & 0.420 \\
\hline 0 vs. III & $2.74(0.34-22.08)$ & 0.340 & $2.22(0.27-18.0)$ & 0.450 \\
\hline \multicolumn{5}{|l|}{ Downstaging } \\
\hline No vs. yes & $0.55(0.19-1.59)$ & 0.270 & - & - \\
\hline \multicolumn{5}{|l|}{$\mathrm{pCR}$} \\
\hline No vs. yes & $0.62(0.08-4.7)$ & 0.640 & - & - \\
\hline \multicolumn{5}{|l|}{ Baseline NLR } \\
\hline$\leq 3.16$ vs. $>3.16$ & $1.8(0.5-6.5)$ & 0.360 & - & - \\
\hline \multicolumn{5}{|l|}{ NLR change (\%) } \\
\hline $\begin{array}{l}-30 \%<\text { NLR change }<100 \% \text { vs. } \\
\text { NLR }<-30 \% \text { or }>100 \%\end{array}$ & $3.81(1.19-12.15)$ & 0.024 & $4.1(1.27-13.16)$ & 0.018 \\
\hline \multicolumn{5}{|l|}{ Ki-67 (\%) } \\
\hline$<20$ vs. $\geq 20$ & $1.29(0.36-4.64)$ & 0.690 & - & - \\
\hline
\end{tabular}

CI, confidence interval; pCR, pathologic complete response; NLR, neutrophil-to-lymphocyte ratio.

\section{Association between survival outcomes and clinico- pathologic features}

For further analysis of the relationship between clinicopathologic parameters and survival outcome, Cox analysis was done. Other than clinical stage, high NLR group was associated with inferior OS and DFS (hazard ratio [HR] for OS, 2.97; $95 \% \mathrm{CI}, 1.43$ to $6.17 ; \mathrm{p}=0.003$ and HR for DFS, 2.11; $95 \% \mathrm{CI}, 1.05$ to $4.25 ; \mathrm{p}=0.036$ ). Multivariate analysis was performed, including clinical stage, Ki-67 index and cancer recurrence during follow-up. Patients with high NLR was still associated with inferior OS (HR, 3.15; 95\% CI, 1.3 to 7.5; $\mathrm{p}=0.009$ ). However, high baseline NLR showed borderline association with inferior DFS (HR, 1.73; 95\% CI, 0.83 to 3.6; $\mathrm{p}=0.140$ ) when considering clinical stage during analysis (Table 3).

Modest NLR change $(-30 \%<$ NLR change $<100 \%)$ during NAC was associated with superior DFS within patients who received NAC (HR, 3.81; 95\% CI, 1.19 to 12.15; $\mathrm{p}=0.024$ ). When adjusted with surgical staging, modest NLR change during NAC was still associated with DFS (HR, 4.1; 95\% CI, 1.27 to $13.16 ; \mathrm{p}=0.018$ ) (Table 4 ).

\section{TILs, PD-L1 expression in tumor tissues and their asso- ciation with NLR}

Total 34 patients had matched baseline tumor tissue and surgical specimen after NAC. The distribution of TILs and PD-L1 expression between baseline tissue and surgical tissue is described in Table 5. Among 34 patients, four patients showed $\mathrm{pCR}$ after neoadjuvant chemotherapy, and 30 surgical specimens were available for analysis. More than half of patients (70\%) showed same or increased TIL after neoadjuvant chemotherapy. Median TIL fold change after NAC was 1.25. In evaluable 30 patients, 12 patients (35.3\%) showed negative tumor cell PD-L1 or negative conversion of PD-L1 expression after NAC. In case of stromal PD-L1 expression, four patients (13.3\%) showed negative PD-L1 or negative conversion of PD-L1 after NAC.

During NAC, patients with radical NLR change showed borderline association to patients who showed negative expression or negative conversion of tumor PD-L1 expression (Fisher exact test, $\mathrm{p}=0.051$ ). The change of NLR after NAC showed negative correlation with the TIL change during NAC, with borderline statistical significance (Fig. 3A). In both baseline tumor tissues and post-NAC tumor tissues, TILs, positive PD-L1 tumor cells, and positive stromal PD-L1 cells showed positive statistical association (Fig. 3B 
Table 5. Distribution of TILs and PD-L1 in tumor tissues

\begin{tabular}{|c|c|c|}
\hline & Baseline tumor tissue & $\begin{array}{l}\text { Tumor tissue after } \\
\text { neoadjuvant chemotherapy }\end{array}$ \\
\hline No. of patients & 34 & 30 \\
\hline Distribution of sTIL & $10(3-35)$ & $11.5(5-60)$ \\
\hline CD4 & $74.6(9.8-226)$ & $67.8(11.6-250.6)$ \\
\hline CD8 & $61.2(6-166.8)$ & $85(19.6-189)$ \\
\hline FOXP3 & $12.4(1.8-54.6)$ & $10.2(1-41)$ \\
\hline CD8/FOXP3 ratio & $4.21(1.2-44.9)$ & $7.66(2.96-26.4)$ \\
\hline \multicolumn{3}{|l|}{ PD-L1 (tumor cell) } \\
\hline Median (range) & $1.5(0-200)$ & $2(0-270)$ \\
\hline$\geq 1$ & $20(58.8)$ & $17(56.7)$ \\
\hline$\geq 5$ & $15(44.1)$ & $14(46.7)$ \\
\hline \multicolumn{3}{|l|}{ PD-L1 (stromal cell) } \\
\hline Median (range) & $8.5(0-90)$ & $17.5(0-125)$ \\
\hline$\geq 1$ & $30(88.2)$ & $24(80.0)$ \\
\hline$\geq 5$ & $21(61.8)$ & $21(70.0)$ \\
\hline \multicolumn{3}{|l|}{ TIL change during NAC } \\
\hline Same or increased & \multicolumn{2}{|r|}{$21(70.0)$} \\
\hline Decreased & \multicolumn{2}{|r|}{$9(30.0)$} \\
\hline \multicolumn{3}{|l|}{ TIL fold change during NAC } \\
\hline Median (range) & \multicolumn{2}{|r|}{$1.25(0.5-8.33)$} \\
\hline \multicolumn{3}{|l|}{ PD-L1 change during NAC } \\
\hline Negative or negative conversion in tumor & \multicolumn{2}{|r|}{$12(35.3)$} \\
\hline Negative or negative conversion in stroma & \multicolumn{2}{|r|}{$4(13.3)$} \\
\hline
\end{tabular}

Value are presented as median (range) or number (\%). TIL, tumor-infiltrating lymphocyte; PD-L1, programmed death-ligand 1; sTIL, stromal TIL; FOXP3, forkhead box P3; NAC, neoadjuvant chemotherapy.

and C).

Immunohistochemical stain for $\mathrm{CD}^{+}, \mathrm{CD}^{+}$, and $\mathrm{FOXP3}^{+}$ TIL was done with paired tissue specimens and the association between each immune-marker stained TIL expression during NAC was analyzed. $\mathrm{CD}^{+}$and $\mathrm{CD}^{+}$was increased in about half of patients ( $48 \%$ and $55.2 \%$, respectively), and $\mathrm{FOXP3}^{+}$was increased in less than half of patients $(34.5 \%)$ (S3 Fig.). There were statistical association between CD4 ${ }^{+}$, $\mathrm{CD}^{+}$, and $\mathrm{FOXP3}^{+}$expression between baseline and postNAC tumor tissues (S4 Fig.).

\section{Survival outcomes according to TIL and PD-L1 expres- sion}

In our study, patients who showed increased TIL during NAC showed trends for favorable DFS, but without statistical significance (Fig. 4A). Patients who showed negative tumor PD-L1 expression, or negative PD-L1 conversion during NAC showed significantly poor survival compared to positive tumor PD-L1 expression during NAC (median DFS, 16.03 months vs. 34.77 months; $\mathrm{p}=0.037$ ) (Fig. 4B). In case of stromal PD-L1 expression, patients showing negative PD-L1 or negative PD-L1 conversion after NAC showed trends for poor DFS, but without statistical significance (Fig. 4C).

When analyzing baseline tumor tissue, positive tumor and stromal PD-L1 expression (PD-L1 H-score $\geq 5$ ) was associated to superior DFS (tumor PD-L1 median DFS, 57.6 months vs. 12.5 months; $\mathrm{p}=0.001$ and stromal PD-L1 median DFS, 50.17 months vs. 20.4 months; $\mathrm{p}=0.002$ ) (Fig. 5A and B). After NAC, patients with positive tumor PD-L1 expression (H-score $\geq 5$ ) showed longer DFS (median DFS, 53.27 months vs. 18.9 months; $p=0.040$ ) (Fig. 5C). In case of stromal PD-L1 expression after NAC, patients with $\mathrm{H}$-score same or higher than 5 showed borderline significance for superior DFS compared to patient with H-score below 5 (median DFS, 38.37 months vs. 17.5 months; $\mathrm{p}=0.063$ ) (Fig. 5D). 
A
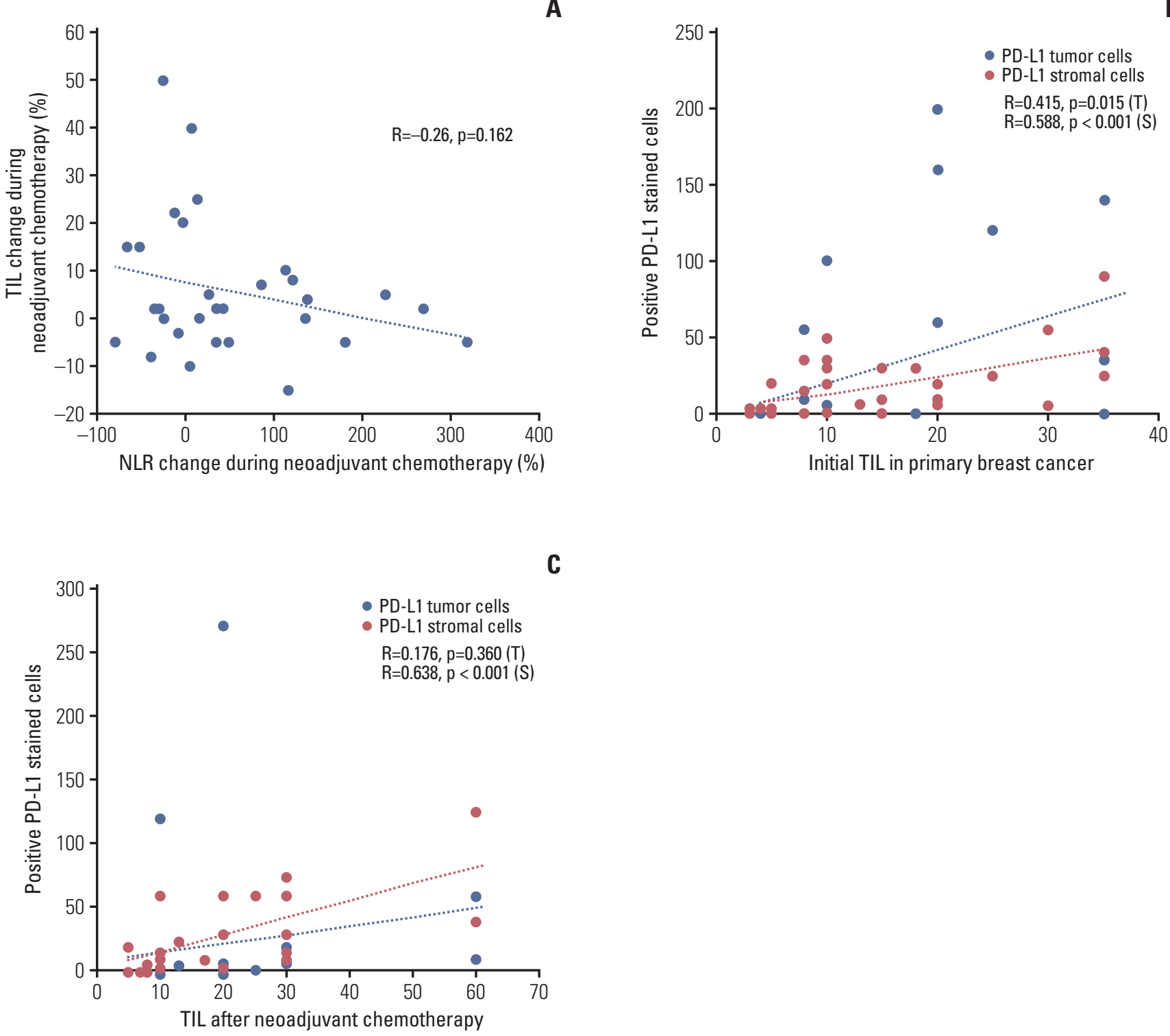

Fig. 3. (A) Correlation between neutrophil-lymphocyte ratio change and tumor infiltrating-lymphocyte (TIL) change during neoadjuvant chemotherapy (NAC). (B) Correlation between TIL and programmed death ligand-1 (PD-L1) changes in primary tumor tissue. (C) Correlation between TIL and PD-L1 changes in post-NAC tissue.

\section{Discussion}

In our study, high baseline NLR was associated to poor survival outcomes in early and advanced TNBC, consistent with previous literatures $[3,5]$. NLR is thought to reflect inflammatory changes during cancer development [3] and has shown relationship with survival outcomes in various solid cancer [24]. There were reports about the role of NLR in TNBC in small population [3], and our study strengthened prior reports with relatively large number of patient population.

Until now, there were scarce reports about the role of changes of serum NLR connected to survival outcome in TNBC during chemotherapy. Considering NLR reflects inflammatory changes and survival outcomes in cancer progression, we hypothesized that changes of NLR during NAC might be associated to cancer response or survival during follow-up. In 50 patients who received NAC, radical changes of NLR (NLR change $<-30 \%$ or $>100 \%$ ) was associated with 

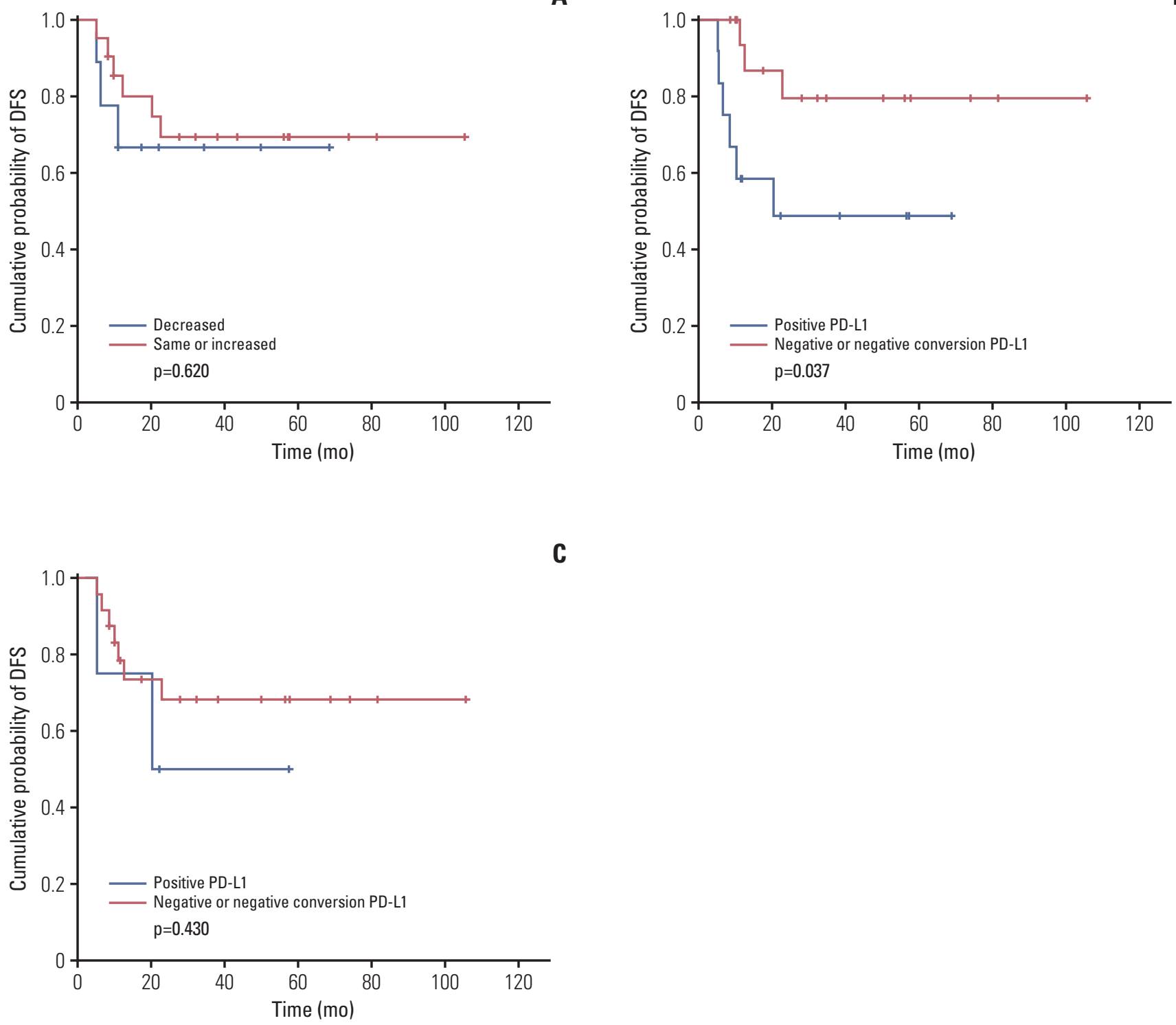

C

Fig. 4. (A) Disease-free survival (DFS) according to changes of tumor infiltrating lymphocyte during neoadjuvant chemotherapy (NAC). (B) DFS according to change of tumor cell programmed death ligand-1 (PD-L1) expression change during NAC. (C) DFS according to change of stromal cell PD-L1 expression during NAC.

inferior DFS. The radical change of NLR was still associated to inferior DFS in multivariate analysis. Changes of NLR during NAC might have reflected cancer-mediated inflammatory changes during treatment [4] and connected to survival and prognosis of breast cancer. We initially assumed that increased NLR after NAC may be associated to poor survival or response, but subtle increase or decrease in serum NLR did not reflect survival outcome during analysis. Radical changes of serum NLR might be associated to definite changes in inflammatory response or changes of cancer environment during treatment, but further analysis should be warranted for confirmation of this hypothesis.

For the first time, we reported the relationship between serum NLR and tumor TIL during NAC. We presumed systemic inflammatory change may be associated to tumor inflammatory change during NAC, and for confirmation of this hypothesis, we analyzed the relationship between changes of serum NLR with tumor TIL change during NAC. Serum NLR and tumor TIL was selected to represent systemic inflammatory changes and local tumor inflammatory 
A
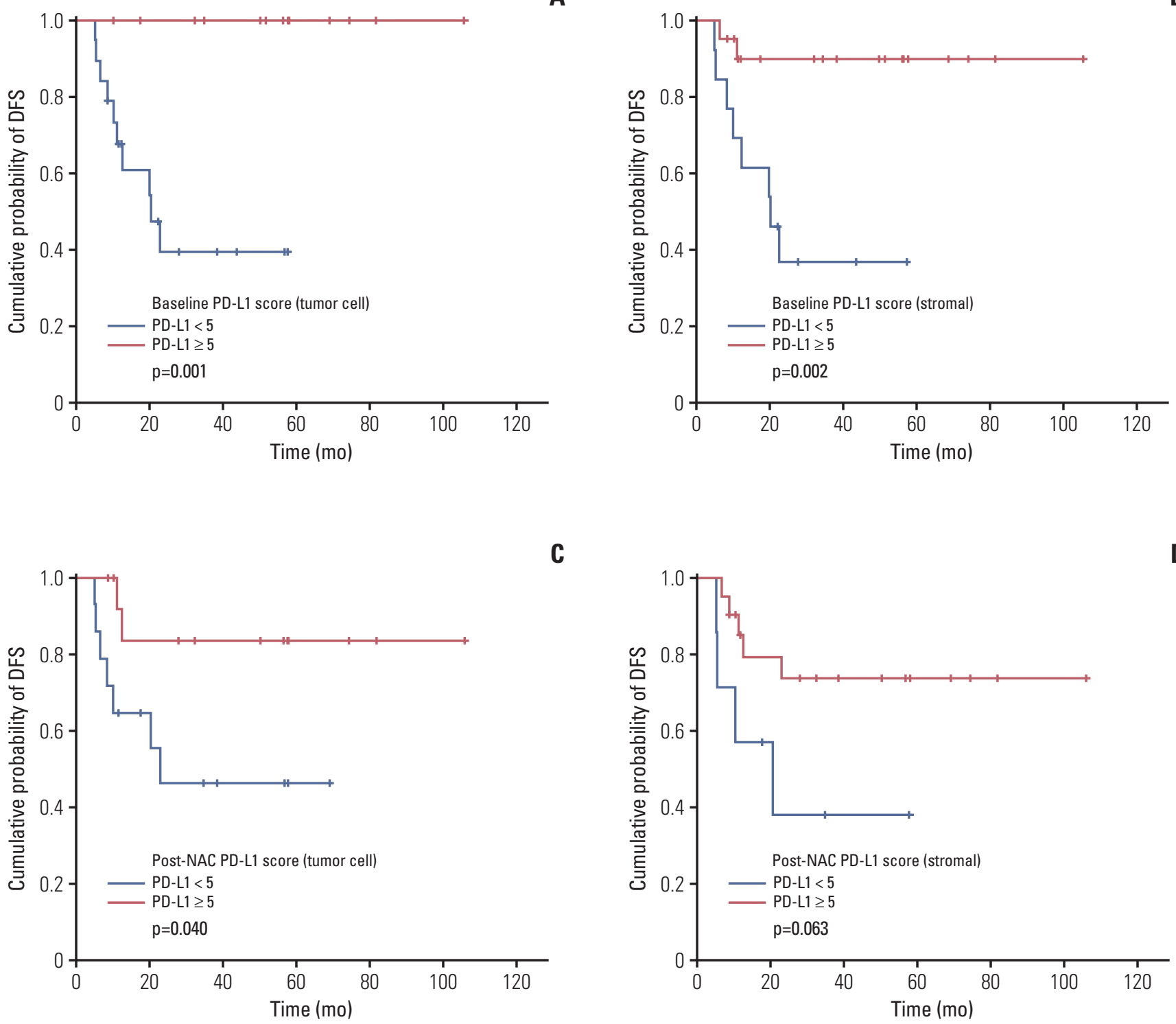

C

Fig. 5. Disease-free survival (DFS) according to tumor cell programmed death ligand-1 expression (PD-L1) (A) and stromal cell PD-L1 expression (B) in baseline tumor tissue. DFS according to tumor cell PD-L1 expression (C) and stromal cell PD-L1 expression (D) in post-neoadjuvant chemotherapy (post-NAC) tumor tissues.

changes, each. There were trends for negative correlation between changes of TIL and serum NLR during NAC, but there was no statistical significance. We assumed other systemic and local inflammatory markers such as cytokines, transcription factors, acute-phase proteins should be considered during interpretation [4], and these confounding factors may have influenced borderline association between serum NLR and tumor TIL.

Previous literature reports the relationship of high pretreatment TIL associated with favorable survival in breast cancer [18], and our result also showed trends for favorable DFS in patients with higher baseline TIL with borderline statistical significance (data not shown). In our study, we investigated the changes between TIL during NAC and analyzed the relationship with survival outcomes. Although the result was not statistically significant, we did find out that there were trends for increased survival in patients with increased or same TIL compared to baseline TIL during NAC. This result is in concordance with previous report [19]. In other retrospective analysis, decreasing TIL was detected after 
NAC and this change was associated to increased pCR rate, but there was no further analysis about accordance to survival outcome with change of TIL [25]. Previous study patient population was heterogeneous in hormonal status and HER2 status, with small patient number. In our study, although we recruited only TNBC patients, but the population was small in number. The change of TIL during NAC seems to influence the tumor response or survival in breast cancer including TNBC, but further study is needed with large number of patients with homogeneous characteristics, and sufficient follow-up duration is needed.

Subtyping of TIL with $\mathrm{CD}^{+}, \mathrm{CD}^{+}$, and $\mathrm{FOXP}^{+}$was done and their association with PD-L1 expression in tumor and stromal TIL was performed in this study. As reported previously, $\mathrm{CD}^{+}, \mathrm{CD}^{+}$, and $\mathrm{FOXP}^{+}$were mutually associated with each other in baseline and post-NAC tissues in our study $[17,26]$. PD-L1 expression in baseline tissue and postNAC tissue was statistically associated to TIL, and this result is consistent with prior study [14]. When TIL was subtyped with $\mathrm{CD}^{+}, \mathrm{CD}^{+}$, or $\mathrm{FOXP}^{+}$, subtyped TIL was partially associated to PD-L1 expression in baseline and post-NAC tissue. $\mathrm{CD}^{+}, \mathrm{CD}^{+}, \mathrm{FOXP3}^{+} \mathrm{TIL}$ all showed trends for positive correlation of PD-L1 expression in tumor tissue. The relationship between TIL subtype and PD-L1 expression showed heterogeneous association among previous reported literatures, with conflicting results in breast cancer (BC) $[17,27,28]$. Persistent, or high level of PD-L1 expression may induced $\mathrm{CD} 8^{+} \mathrm{T}$ cell exhaustion, and also may be related to $\mathrm{CD} 4^{+}$or $\mathrm{FOXP3}^{+} \mathrm{T}$ cells which maintain immune response and expression of PD-L1 expression [29]. Considering there are negative relationship between regulatory $\mathrm{T}$ cell, effector $\mathrm{T}$ cell and PD-L1 expression, negative correlation between TIL subtype, especially CD8 ${ }^{+}$TIL and PD-L1 might be estimated, and there are reports supporting this view $[17,30]$. However, there are other reports supporting $\mathrm{CD} 8^{+} \mathrm{T}$ cell may recruit $\mathrm{CD}^{+}$or $\mathrm{FOXP}^{+}$regulatory $\mathrm{T}$ cells with PD-L1 by intrinsic immune mechanism [31], and this hypothesis is in consistence to our result. When interpreting the relationship between TIL, TIL subtype and PD-L1 expression, various tumor induced environmental change and immune mechanism may influence the expression of T cell and PD-L1 expression, and this might have influenced the conflicting reports about the TIL subtype and PD-L1 expression in BC.

As one of an immune modulatory marker and representing tumor microenvironment [14], the change of PD-L1 expression during NAC was analyzed in the study. Patients who showed negative tumor PD-L1 or negative conversion of tumor PD-L1 expression during NAC showed poor outcome. Furthermore, low PD-L1 expression in tumor or stromal tissue $(\mathrm{H}$-score $<5)$ in baseline tumor or post-NAC tissue also showed poor DFS. To date, the role of PD-L1 expression and its association to clinical outcome is controversial. Part or studies report increased PD-L1 expression is associated to poor prognosis or aggressive clinical behavior in $\mathrm{BC}$ or other cancers $[15,17,21,30]$, and there are other studies reporting PD-L1 expression associated to favorable outcome in solid cancers, consistent with our analysis $[14,20,32]$. This discrepancy may arise from the heterogeneous composition of various solid tumors in the study, and various definition of PD-L1 status among each study may have influenced the conflicting results of previous reports as well. Furthermore, different molecular profile and oncogenic mechanism between $\mathrm{BC}$ subtypes or other types of cancer may have influenced the role of PD-L1, which might have relatively different role between various cancers and different breast cancer subtypes. The merit of our study lies on relatively homogenous patient population, consisted of locally advanced TNBC treated with similar systemic chemotherapy. Considering TNBC is more immunogenic compared to other subtypes, PD-L1 and its associated molecular pathway may have different role compared to other BC subtypes.

In present study, there are some limitations to mention. First, we included TNBC patients diagnosed and treated until Dec 2015. We included recently diagnosed TNBC patients with adequate archival tumor tissue, but the followup duration was relatively short compared to other studies. Long-term follow up of the results of our study is needed for future study. Second, as we mentioned, although the subtype was homogeneous, the patient population with adequate tumor tissue was relatively small, requiring careful interpretation of the analysis. However, this study has its clinical value for selecting only TNBC patients with paired tumor samples. Based on our analysis, we are planning to review the change of NLR, TIL, and PD-L1 expression in large number of scale, comprising multicenter in Korea. Sufficient follow-up duration with large number of patient population is warranted for future study.

In conclusion, this study showed high baseline NLR associated to poor survival outcome in locally advanced TNBC patients. Among patients receiving NAC, radical change of serum NLR during chemotherapy was associated to poor prognosis. Increased TIL in post-NAC tumor tissue showed trends for superior DFS during follow-up, but it did not reach statistical significance during analysis. Finally, negative conversion of PD-L1 expression or persistent negative expression of PD-L1 in tumor tissue during NAC or low level of PD-L1 expression in baseline or post-NAC tumor tissue was associated to poor prognosis. For more confirmative results, larger sample size of patients with sufficient followup duration is warranted. 
Electronic Supplementary Material

Supplementary materials are available at Cancer Research and Treatment website (https:// www.e-crt.org).

\section{Conflicts of Interest}

Conflict of interest relevant to this article was not reported.

\section{Acknowledgments}

The present research has been supported by Korea Breast Cancer Foundation and Basic Science Research Program through the National Research Foundation of Korea funded by the Ministry of Science, ICT and Future Planning (NRF-2017R1D1A1B03034165).

\section{References}

1. Malorni L, Shetty PB, De Angelis C, Hilsenbeck S, Rimawi MF, Elledge $\mathrm{R}$, et al. Clinical and biologic features of triple-negative breast cancers in a large cohort of patients with long-term follow-up. Breast Cancer Res Treat. 2012;136:795-804.

2. Lehmann BD, Bauer JA, Chen X, Sanders ME, Chakravarthy $A B$, Shyr $Y$, et al. Identification of human triple-negative breast cancer subtypes and preclinical models for selection of targeted therapies. J Clin Invest. 2011;121:2750-67.

3. Pistelli M, De Lisa M, Ballatore Z, Caramanti M, Pagliacci A, Battelli N, et al. Pre-treatment neutrophil to lymphocyte ratio may be a useful tool in predicting survival in early triple negative breast cancer patients. BMC Cancer. 2015;15:195.

4. Diakos CI, Charles KA, McMillan DC, Clarke SJ. Cancer-related inflammation and treatment effectiveness. Lancet Oncol. 2014;15:e493-503.

5. Orditura M, Galizia G, Diana A, Saccone C, Cobellis L, Ventriglia J, et al. Neutrophil to lymphocyte ratio (NLR) for prediction of distant metastasis-free survival (DMFS) in early breast cancer: a propensity score-matched analysis. ESMO Open. 2016;1:e000038.

6. Azab B, Bhatt VR, Phookan J, Murukutla S, Kohn N, Terjanian $\mathrm{T}$, et al. Usefulness of the neutrophil-to-lymphocyte ratio in predicting short- and long-term mortality in breast cancer patients. Ann Surg Oncol. 2012;19:217-24.

7. Chen Y, Chen K, Xiao X, Nie Y, Qu S, Gong C, et al. Pretreatment neutrophil-to-lymphocyte ratio is correlated with response to neoadjuvant chemotherapy as an independent prognostic indicator in breast cancer patients: a retrospective study. BMC Cancer. 2016;16:320

8. Loi S, Sirtaine N, Piette F, Salgado R, Viale G, Van Eenoo F, et al. Prognostic and predictive value of tumor-infiltrating lymphocytes in a phase III randomized adjuvant breast cancer trial in node-positive breast cancer comparing the addition of docetaxel to doxorubicin with doxorubicin-based chemotherapy: BIG 02-98. J Clin Oncol. 2013;31:860-7.

9. Zhang L, Conejo-Garcia JR, Katsaros D, Gimotty PA, Massobrio M, Regnani G, et al. Intratumoral T cells, recurrence, and survival in epithelial ovarian cancer. N Engl J Med. 2003;348: 203-13.

10. Galon J, Costes A, Sanchez-Cabo F, Kirilovsky A, Mlecnik B, Lagorce-Pages $\mathrm{C}$, et al. Type, density, and location of immune cells within human colorectal tumors predict clinical outcome. Science. 2006;313:1960-4.

11. Miyashita M, Sasano H, Tamaki K, Hirakawa H, Takahashi Y, Nakagawa S, et al. Prognostic significance of tumor-infiltrating $\mathrm{CD} 8+$ and FOXP3+ lymphocytes in residual tumors and alterations in these parameters after neoadjuvant chemotherapy in triple-negative breast cancer: a retrospective multicenter study. Breast Cancer Res. 2015;17:124.

12. Chen DS, Irving BA, Hodi FS. Molecular pathways: next-generation immunotherapy: inhibiting programmed death-ligand 1 and programmed death-1. Clin Cancer Res. 2012;18:6580-7.

13. Patel SP, Kurzrock R. PD-L1 expression as a predictive biomarker in cancer immunotherapy. Mol Cancer Ther. 2015;14: 847-56.

14. Wimberly H, Brown JR, Schalper K, Haack H, Silver MR, Nixon C, et al. PD-L1 expression correlates with tumor-infiltrating lymphocytes and response to neoadjuvant chemotherapy in breast cancer. Cancer Immunol Res. 2015;3:326-32.

15. Muenst S, Schaerli AR, Gao F, Daster S, Trella E, Droeser RA, et al. Expression of programmed death ligand 1 (PD-L1) is associated with poor prognosis in human breast cancer. Breast Cancer Res Treat. 2014;146:15-24.

16. Sabatier R, Finetti P, Mamessier E, Adelaide J, Chaffanet M, Ali HR, et al. Prognostic and predictive value of PDL1 expression in breast cancer. Oncotarget. 2015;6:5449-64.

17. Park IH, Kong SY, Ro JY, Kwon Y, Kang JH, Mo HJ, et al. Prognostic implications of tumor-infiltrating lymphocytes in association with programmed death ligand 1 expression in earlystage breast cancer. Clin Breast Cancer. 2016;16:51-8.

18. Pruneri G, Vingiani A, Bagnardi V, Rotmensz N, De Rose A, Palazzo A, et al. Clinical validity of tumor-infiltrating lymphocytes analysis in patients with triple-negative breast cancer. Ann Oncol. 2016;27:249-56.

19. Pelekanou V, Carvajal-Hausdorf DE, Altan M, Wasserman B, Carvajal-Hausdorf C, Wimberly $\mathrm{H}$, et al. Effect of neoadjuvant chemotherapy on tumor-infiltrating lymphocytes and PD-L1 expression in breast cancer and its clinical significance. Breast Cancer Res. 2017;19:91.

20. Li X, Wetherilt CS, Krishnamurti U, Yang J, Ma Y, Styblo TM, et al. Stromal PD-L1 expression is associated with better disease-free survival in triple-negative breast cancer. Am J Clin 
Pathol. 2016;146:496-502.

21. Muenst S, Soysal SD, Gao F, Obermann EC, Oertli D, Gillanders WE. The presence of programmed death 1 (PD-1)-positive tumor-infiltrating lymphocytes is associated with poor prognosis in human breast cancer. Breast Cancer Res Treat. 2013; 139:667-76.

22. Hammond ME, Hayes DF, Dowsett M, Allred DC, Hagerty KL, Badve S, et al. American Society of Clinical Oncology / College of American Pathologists guideline recommendations for immunohistochemical testing of estrogen and progesterone receptors in breast cancer (unabridged version). Arch Pathol Lab Med. 2010;134:e48-72.

23. Wolff AC, Hammond ME, Hicks DG, Dowsett M, McShane LM, Allison KH, et al. Recommendations for human epidermal growth factor receptor 2 testing in breast cancer: American Society of Clinical Oncology/College of American Pathologists clinical practice guideline update. Arch Pathol Lab Med. 2014;138:241-56.

24. Guthrie GJ, Charles KA, Roxburgh CS, Horgan PG, McMillan DC, Clarke SJ. The systemic inflammation-based neutrophillymphocyte ratio: experience in patients with cancer. Crit Rev Oncol Hematol. 2013;88:218-30.

25. Pelekanou V, Barlow WE, von Wahlde MK, Wasserman B, Lo YC, Hayes DF, et al. Effects of neoadjuvant chemotherapy (NAC) on tumor infiltrating lymphocytes (TIL) and PD-L1 expression in the SWOG S0800 clinical trial. J Clin Oncol. 2017;35:15 Suppl:519.

26. Seo AN, Lee HJ, Kim EJ, Kim HJ, Jang MH, Lee HE, et al. Tumour-infiltrating CD8+ lymphocytes as an independent predictive factor for pathological complete response to primary systemic therapy in breast cancer. Br J Cancer. 2013;109: 2705-13.

27. Cimino-Mathews A, Thompson E, Taube JM, Ye X, Lu Y, Meeker A, et al. PD-L1 (B7-H1) expression and the immune tumor microenvironment in primary and metastatic breast carcinomas. Hum Pathol. 2016;47:52-63.

28. Ghebeh H, Barhoush E, Tulbah A, Elkum N, Al-Tweigeri T, Dermime S. FOXP3+ Tregs and B7-H1+ / PD-1+ T lymphocytes co-infiltrate the tumor tissues of high-risk breast cancer patients: Implication for immunotherapy. BMC Cancer. 2008; 8:57.

29. Ohaegbulam KC, Assal A, Lazar-Molnar E, Yao Y, Zang X. Human cancer immunotherapy with antibodies to the PD-1 and PD-L1 pathway. Trends Mol Med. 2015;21:24-33.

30. Hamanishi J, Mandai M, Iwasaki M, Okazaki T, Tanaka Y, Yamaguchi K, et al. Programmed cell death 1 ligand 1 and tumor-infiltrating CD8+ T lymphocytes are prognostic factors of human ovarian cancer. Proc Natl Acad Sci U S A. 2007;104: 3360-5.

31. Spranger S, Spaapen RM, Zha Y, Williams J, Meng Y, Ha TT, et al. Up-regulation of PD-L1, IDO, and T(regs) in the melanoma tumor microenvironment is driven by CD8(+) T cells. Sci Transl Med. 2013;5:200ra116.

32. Badoual C, Hans S, Merillon N, Van Ryswick C, Ravel P, Benhamouda $\mathrm{N}$, et al. PD-1-expressing tumor-infiltrating T cells are a favorable prognostic biomarker in HPV-associated head and neck cancer. Cancer Res. 2013;73:128-38. 